University of Louisville

ThinkIR: The University of Louisville's Institutional Repository

\title{
Investigating the mechanism of novel anticancer agent, AS1411 : does metabolism to guanine play a role?
}

\author{
Parker T Howard \\ University of Louisville
}

Follow this and additional works at: https://ir.library.louisville.edu/honors

Part of the Biochemical Phenomena, Metabolism, and Nutrition Commons, Medical Biochemistry Commons, and the Nucleic Acids, Nucleotides, and Nucleosides Commons

\section{Recommended Citation}

Howard, Parker T, "Investigating the mechanism of novel anticancer agent, AS1411 : does metabolism to guanine play a role?" (2017). College of Arts \& Sciences Senior Honors Theses. Paper 147.

http://doi.org/10.18297/honors/147

This Senior Honors Thesis is brought to you for free and open access by the College of Arts \& Sciences at ThinkIR: The University of Louisville's Institutional Repository. It has been accepted for inclusion in College of Arts \& Sciences Senior Honors Theses by an authorized administrator of ThinkIR: The University of Louisville's Institutional Repository. This title appears here courtesy of the author, who has retained all other copyrights. For more information, please contact thinkir@louisville.edu. 
Investigating the Mechanism of Novel Anticancer Agent, AS1411: Does Metabolism to Guanine Play a Role?

\author{
By \\ Parker Thomas Howard
}

Submitted in partial fulfillment of the requirements

for Graduation summa cum laude

and

for Graduation with Honors from the Department of Biology

University of Louisville

$3 / 24 / 2017$ 


\section{Background}

Despite the ongoing developments made in modern medical research, cancer remains the second most common cause of death in the United States [1]. Many modern treatments, such as radiation therapy and chemotherapy, have numerous adverse side-effects that may not become evident until months or years after treatment has ended [2 \& 3]. Additionally, there exist few effective treatments for individuals diagnosed with late-stage cancers. There is a great need for continued research and development of novel treatment options to facilitate better clinical outcomes of cancer patients.

Our research of the drug AS1411 aims to address this need for improved cancer therapeutics. AS1411 is a guanine-rich oligonucleotide optimized for its anti-proliferative activity of cancer cells. Guanine-rich oligonucleotides (GROs) are short chains of

nucleotides ranging from

approximately 13 to 25

nucleotides and are

predominately composed of

guanine residues [4]. GROs

exhibit a number of properties

that amplify their activity,

including spontaneously

forming G-quadruplex

structures (Figure 1) which

increase the compound's
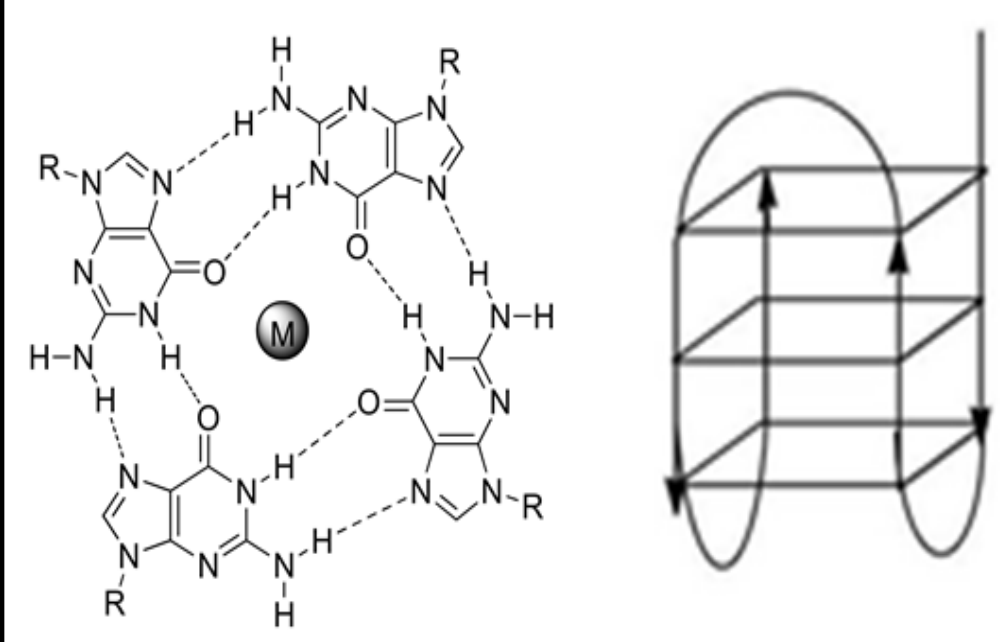

Figure 1. An example structure of a G-quadruplex in two views. (Left) Demonstrates guanine residues forming hydrogen bonds in the presence of a cation to create a stable quadruplex. (Right) Illustrates how the backbone of the oligonucleotide can loop into a stable conformation. Adapted from Ma et al. 2013 [7]. 
ability to resist degradation by nucleases [5]. While the exact quadruplex structure of AS1411is unknown, at least 8 different forms have been detected using a size exclusion chromatography [6]. Additionally, AS1411 has specificity for cancer cells because it is capable of binding to nucleolin, a protein that is highly expressed on the surface on many types of cancer cells [8].

AS1411 has shown promise in both in vitro and in vivo experiments. The $\mathrm{Gl}_{50}$ values (the concentration required for $50 \%$ growth inhibition) for AS1411 were remarkably low for almost every tested cancer cell line despite the fact of having little effect in normal cell lines at similar concentrations [5]. Notably, the compound also inhibited tumor growth in mouse models despite displaying no toxicity in normal tissues. These results imply that the anti-proliferative effects of AS1411 selectively target cancerous cells, unlike standard therapies. In 2003, AS1411 entered into a Phase 1 clinical trial in patients with advanced cancers. During this trial, there were no reports of severe side-effects and varying degrees of clinical activity. Most patients experienced disease stabilization, but one patient experienced a complete response; within 11 months [5]. Similar results were later replicated during phase 2 clinical trials, however the funding company, Antisoma, had to terminate ongoing trials due to the expensive failure of another drug compound in its pipeline [8]. AS1411 was tested in over 100 patients but only demonstrated strong activity in 7 . However, these 7 patients exhibited strong results including substantial shrinkage and the complete disappearance of tumors [9]. It was discerned that in order to apply and optimize AS1411, a better understanding of the mechanism of action was required [ $8 \& 9]$. 
AS1411 is incorporated into the cell by macropinocytosis and that levels of macropinocytosis and anti-proliferative activity are correlated [10]. In particular, AS1411 induces cell death through a novel pathway known as methuosis, in which the cells ingest numerous vesicles causing eventual cell lysis [10 \& 11]. Methuosis is a novel nonapoptotic cell death pathway that is characterized by displacement of the cytoplasm by large fluid-filled vacuoles derived from macropinosomes that ultimately induce cell lysis. Notably, this pathway involves the hyperstimulation of a number of oncogenic pathways that ultimately result in cell death [12]. The involvement of these oncogenic pathways makes methuosis highly promising as a possible target for the treatment of aggressive or late-stage disease. Although numerous compounds and proteins have been implicated in the pathway, many aspects of the pathway remain unknown.

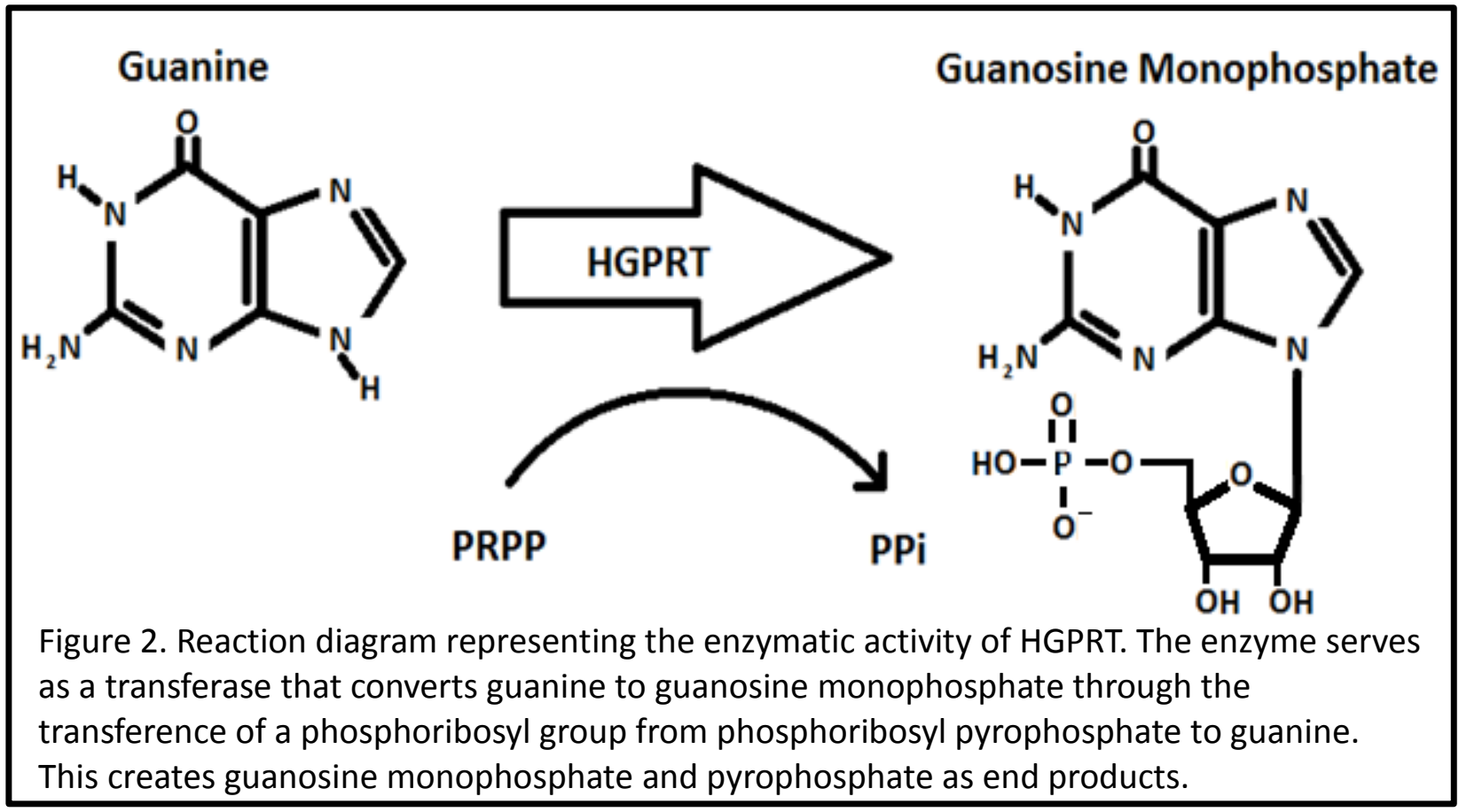

The accumulation of guanine ribonucleotides can lead to the arrest of the cell cycle and an inhibition of cellular growth in human cells [13]. Further studies have 
suggested that the degradation of GROs (including AS1411) to the monomers of guanine-based purine compounds (GBPCs) such as guanine, guanosine, and guanosine monophosphate dictate the anti-proliferative effects in vitro [14]. Additionally, these anti-proliferative effects are dependent on hypoxanthine-guanine phosphoribosyltransferase (HGPRT), an enzyme in the purine salvage pathway (Figure 2) [15]. HGPRT is a vital element of the purine salvage pathway and subsequently an important enzyme for creating the bases that will be sequestered into new DNA [16]. A loss of HGPRT function in cells makes them unable to recycle certain purines, and this causes deleterious effects.

This can be seen in the

severely shortened lifespans

of patients affected by Lesch-

Nyhan syndrome, an inherited disorder caused by an HGPRT deficiency [17]. My current hypothesis (Figure 3) posits that AS1411 acts as a prodrug for guanine and that the antiproliferative activity should therefore be dependent upon the activity of HGPRT. Some studies in the Bates lab have indicated that metabolism to

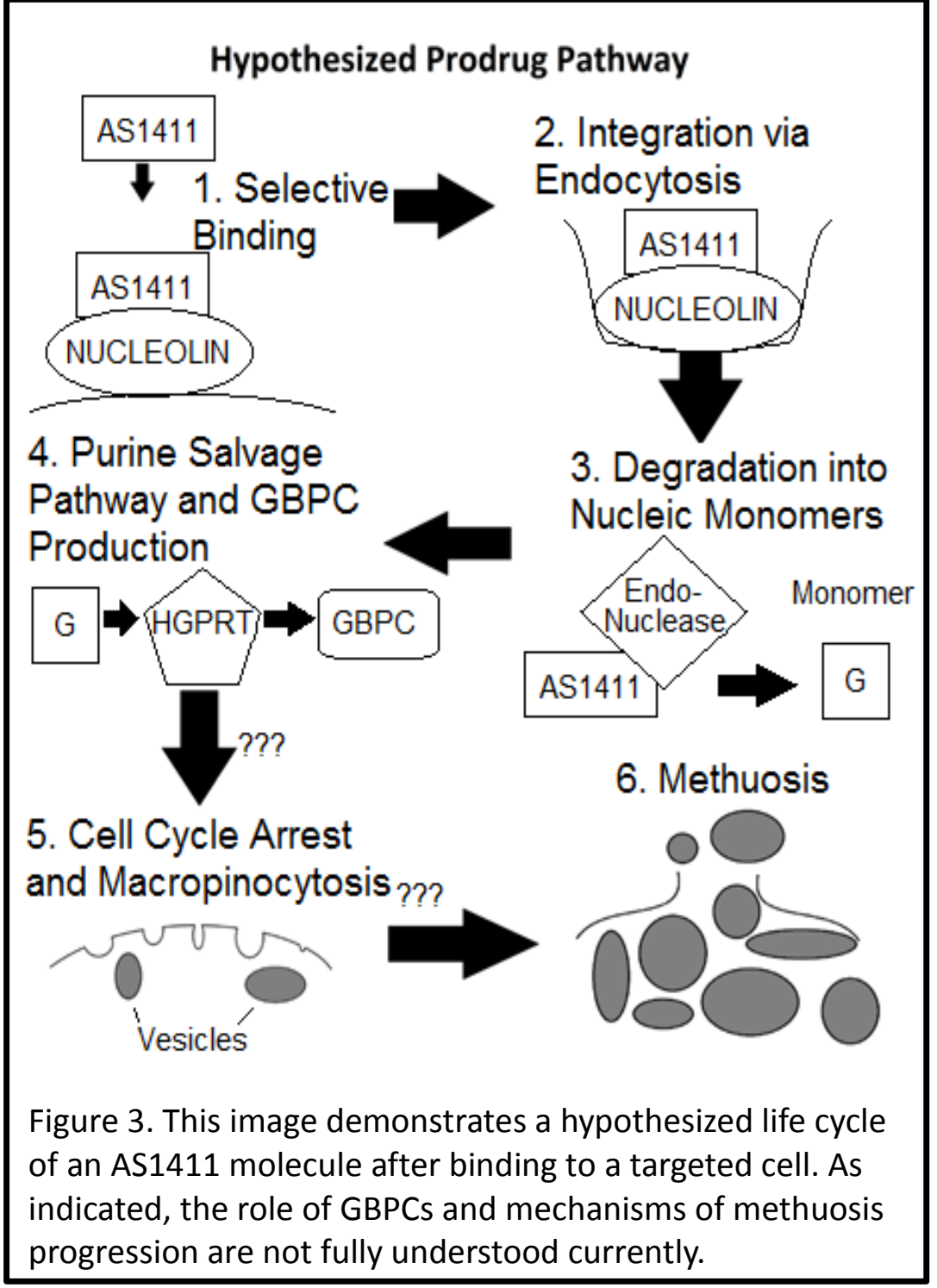


guanine plays a role in AS1411 activity [unpublished]; however, more research is needed to elucidate the mechanism. This project will be combined with our previous unpublished research with the goal of definitively testing the stated hypothesis. Ultimately, this knowledge will serve to guide further studies for optimizing AS1411 as a potential cancer treatment.

\section{$\underline{\text { Specific Aims }}$}

The specific aim for this project was to investigate the possible role of HGPRT in the anti-proliferative activity of AS1411. If degradation to GBPCs is vital for AS1411 activity, then the loss of HGPRT function should result in a decrease of the compounds exhibited anti-proliferative effects.

\section{Materials and Methods}

\section{Materials}

Oligodeoxynucleotides, AS1411 (5'-GGTGGTGGTGGTTGTGGTGGTGGTGG), and negative control oligonucleotide, CRO (5'-ССTCCTCCTCCTTCTCCTCCTCCTCC) were purchased in the desalted form from Integrated DNA Technologies (Coralville, IA). Guanosine and 6-thioguanine were purchased from Sigma (St. Louis, MO). Mouse monoclonal antibodies, $\alpha$-tubulin (11H10, Cell Signaling, Danvers, MA) and HGPRT (F1, cat \# SC-376938,Santa Cruz Biotech, Dallas, TX) were utilized along with anti-mouse (sc-2005, Santa Cruz Biotech) and anti-rabbit (sc-2004, Santa Cruz Biotech) antibodies linked to horseradish peroxidase. Small interfering ribonucleic acids (siRNAs) targeting human HGPRT, s6887 (5'-GGAUAUGCCCUUGACUAUAtt-3'), s6888 (5'- 
AAAUAGUGAUAGAUCCAtt-3'), and Silencer Select Negative Control \#1 were purchased from Life Technologies (Carlsbad, CA).

\section{Cell Lines}

A549 cell lines were purchased from the American Type Culture Collection (ATCC) and grown in Dulbecco's Modified Eagle's medium (DMEM) media (Life Technologies) supplemented with 1\% penicillin/streptomycin (Life Technologies) and $10 \%$ fetal bovine serum (Life Technologies) in humidified incubators at $37^{\circ} \mathrm{C}$ with $5 \%$ $\mathrm{CO}_{2}$. Cells were plated and incubated overnight to allow for adherence prior to treatment.

\section{Cell Lysis and Protein Collection}

Flasks containing cells were placed on ice and media was removed. Flasks were then washed with ice-chilled phosphate-buffered saline (PBS, Life Technologies). Cells were lysed in RIPA buffer [150 mmol/L NaCl, $2 \mathrm{mmol} / \mathrm{L}$ EDTA, $50 \mathrm{mmol} / \mathrm{L}$ Tris-HCl, $0.25 \%$ deoxycholic acid, $1 \%$ IGEPAL CA-630 (pH 7.5)] containing protease and phosphatase inhibitors (Calbiotech, Spring Valley, CA) and collected using a cell scraper (TPP, Trasadingen, Switzerland) and clarified by centrifugation at $14,000 \times g$ for 10 min at $4{ }^{\circ} \mathrm{C}$. Diluted clarified total cell lysates were analyzed using a Pierce ${ }^{\mathrm{TM}} \mathrm{BCA}$ Protein assay kit against a standardized protein concentration curve derived from known bovine serum albumin (BSA) standards (Fisher Scientific, Waltham, MA).

\section{Protein Analysis by Western Blot}


Samples for SDS-PAGE were prepared using $25 \mu \mathrm{g}$ of protein, $4 \times$ loading buffer containing $10 \% \beta$-mercaptoethanol, and distilled water to ensure samples of equal volumes. Samples were resolved using Novex $4-20 \%$ tris-gylcine gels (Life Technologies) and then transferred onto polyvinylidine fluoride membranes (Fisher Scientific) in Tris-glycine transfer buffer (Life Technologies) containing 20\% methanol. Membranes were either blocked with $5 \%$ milk in tris-buffered saline containing $0.05 \%$ tween-20 (TBS-T) in TBS-T. The following primary concentrations were used for protein detection: HGPRT 1:500, and a-tubulin 1:1000. Secondary antibodies were used at a 1:10,000 concentration. Membranes probed with HGPRT primaries were detected using SuperSignal® West Dura ECL (Fisher Scientific) while $\alpha$-tubulin was detected using Pierce ${ }^{\circledR}$ ECL Western Blotting Substrate (Fisher Scientific). Chemiluminescence was visualized using Amersham Hyperfilm ${ }^{\mathrm{TM}}$ (GE Healthcare, Little Chalfont, United Kingdom).

\section{Cell Proliferation Assay}

A549 cells were plated at a density of 1000 cells per well in 96 well plates (TPP) and incubated overnight to allow for adherence. Cells were treated in triplicate wells with either oligonucleotide, GBPC, or siRNAs. After treatments of varying duration, MTT (Sigma) was added in the dark at 1/10th total sample volume, and cells were incubated for 4 hours. Lysis buffer (10\% SDS in $0.01 \mathrm{~N} \mathrm{HCl})$ was added at half of the original sample volume and incubated overnight to ensure complete cell lysis and dissolution of crystals. Plates were read at $570 \mathrm{~nm}$, and relative absorbance values were exported to Microsoft Exce ${ }^{\circledR}$ for further analysis [18 \& 19]. GraphPad Prism was used to determine the level of statistical significance by ANOVA and/or t-test. 


\section{HGPRT Knockdown via siRNA Transfection for Protein Analysis}

A549 cells were transfected with Lipofectamine 2000 (Intivrogen, Carlsbad, CA) and Opti-MEM ${ }^{\circledR}$ (Life Technologies) per manufacturer's protocol. Briefly, cells were transferred to antibiotic free media for 1 hour before transfection. Cells were then either transfected with HGPRT siRNAs (s6887 \& s6888), or negative control \#1 siRNA (Life Technologies) as seen in Figure 4. After 4 hours, the media containing transfected reagents was replaced with complete media. Transfected cells were evaluated for relative proliferation levels using MTT colorimetric assays or lysed for analysis of protein levels using western blots.

\section{Example Plate Layout for Transfection and Treatment}

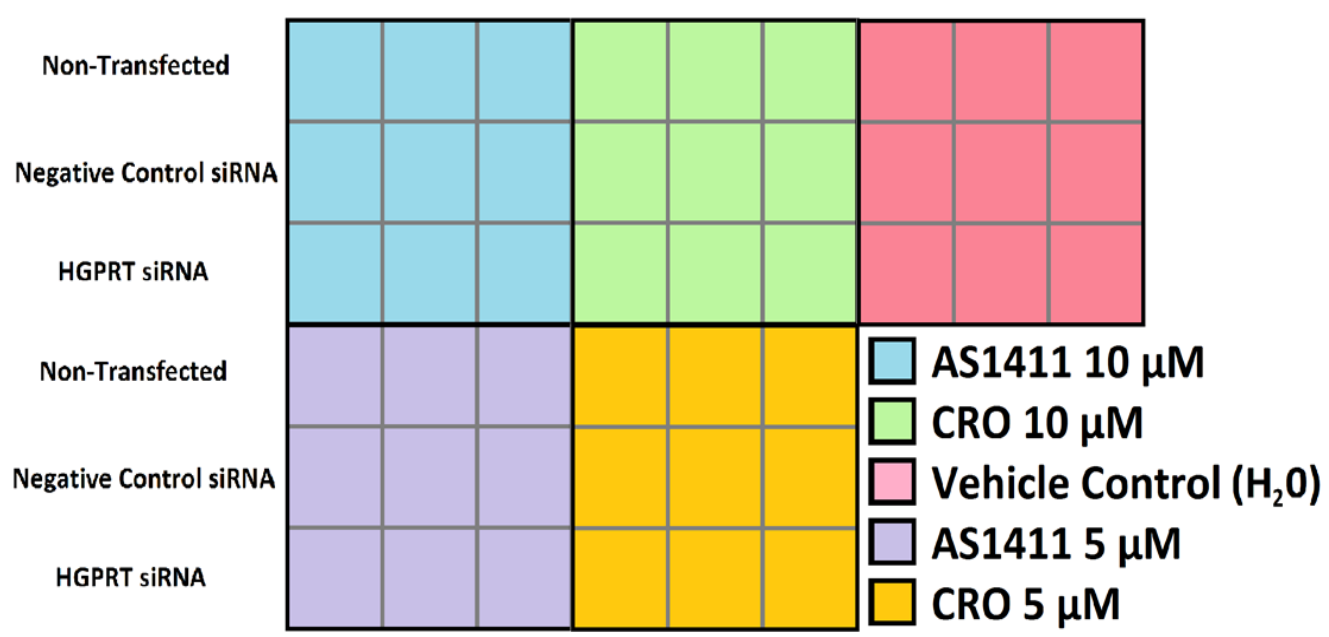

Figure 4. Example plate demonstrating how treatment groups are further divided by transfection type. Each combination of treatment and transfection is represented by an average of three individual wells. 


\section{Development of Thioguanine-Resistant A549 Cells}

A549 cells were grown to $~ 70 \%$ confluency and then transferred to new flasks containing a $1.5 \mu \mathrm{M}$ 6-thioguanine (Sigma). After cells grew to confluency, they were passaged into new flasks with $3 \mu \mathrm{M}$ increases in concentration of 6 -thioguanine. This was repeated until final concentrations of $6 \mu \mathrm{M}$ and $15 \mu \mathrm{M}$ of 6 -thioguanine were established.

$\underline{\text { Results }}$

Determining Optimal Conditions for AS1411 Anti-Proliferative Effects

In an effort to

identify the

concentration of

AS1411 necessary

for evident anti-

proliferative activity

in A549 lines, A549

cells were plated at

a density of 1000

cells per well in 96

well plates and

incubated in
Effect of AS1411 on A549 Cell Proliferation

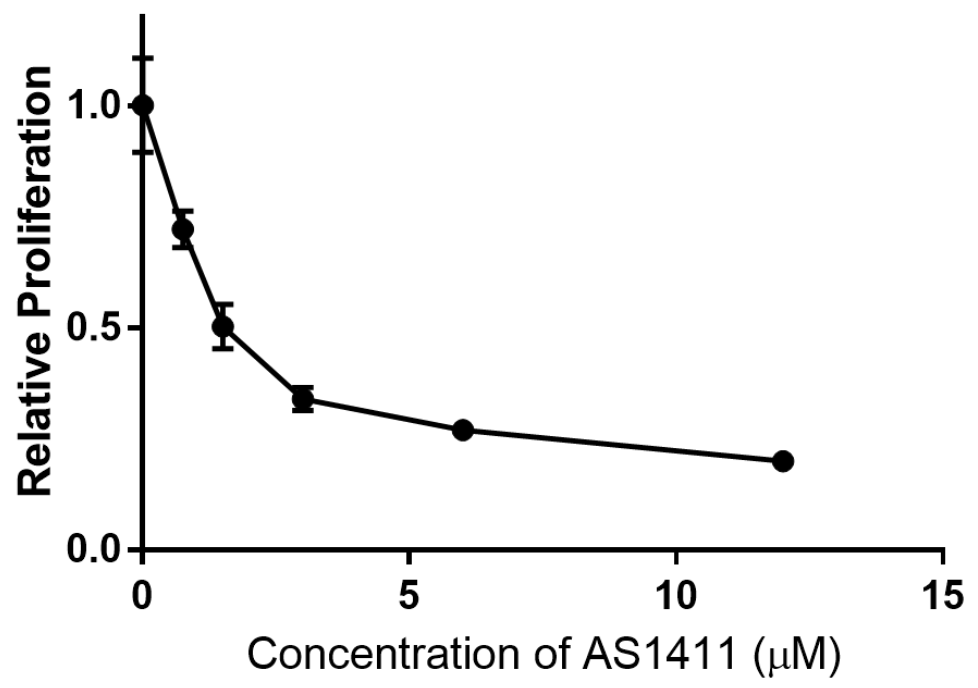

Figure 5. Relative proliferation levels of A549 cells treated with increasing concentrations of AS1411. Results from MTT colorimetric assay were normalized against a vehicle control. Error bars represent one standard deviation from the mean.

complete media overnight to allow for adherence. Cells were then treated in triplicate wells with increasing concentrations of AS1411 and incubated for 72 hours before cell 
proliferation assay were performed. Results were normalized to a vehicle control.

Substantial decreases in relative proliferation were observed with a Gl 50 value of approximately $1.5 \mu \mathrm{M}$ AS1411 (Figure 5).

\section{Determining Optimal Conditions for HGPRT Knockdown using siRNAs}

A549 cell lines were treated with HGPRT siRNAs to knockdown protein expression, and subsequent protein samples were analyzed by western blot to confirm knockdown. Initially, A549 cells were transfected with HGPRT siRNAs (s6887 \& s6888), a negative control siRNA, or left untransfected. After 48 hours, the cells were lysed and

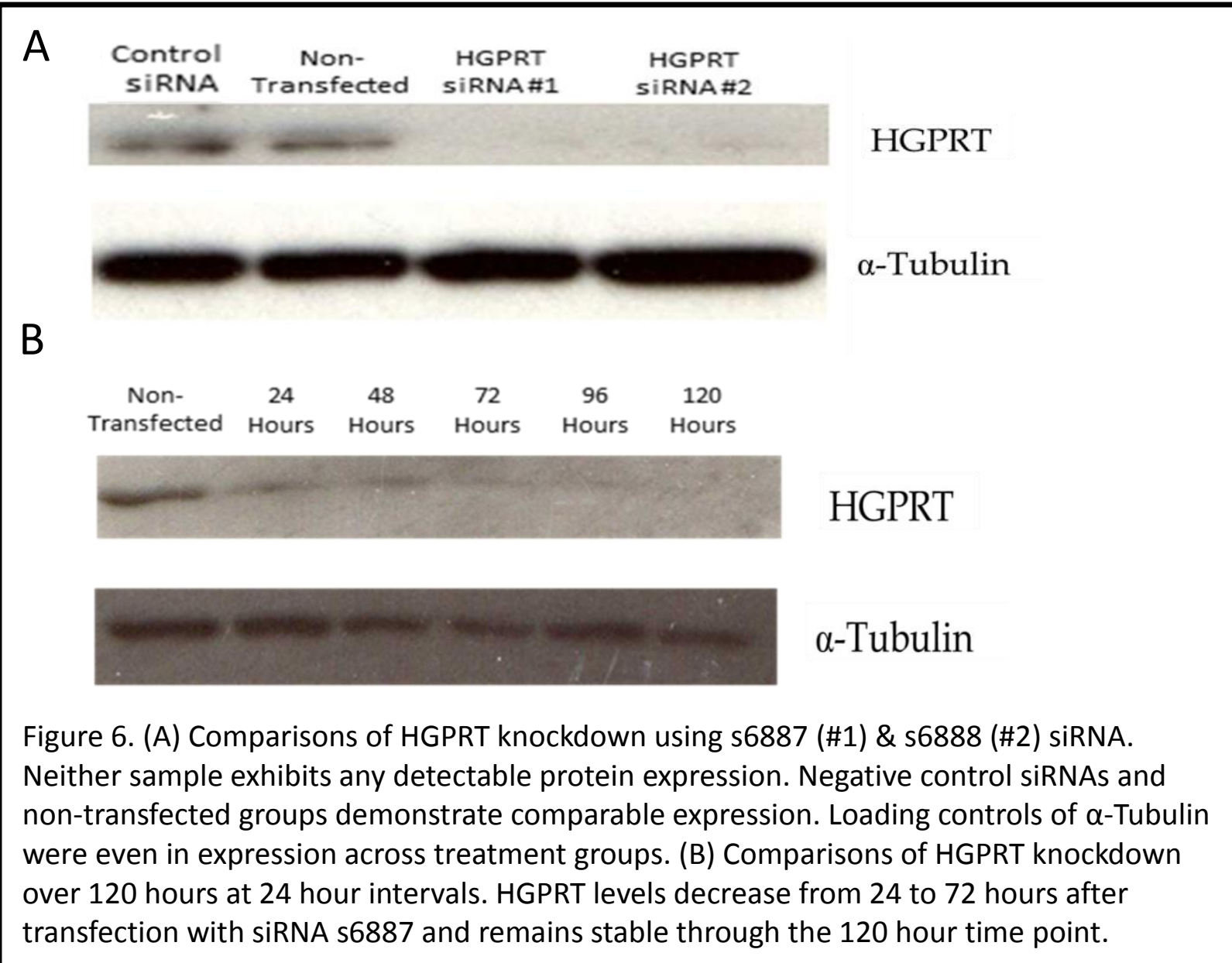


HGPRT levels were determined by western blot. $\alpha$-Tubulin was also examined to ensure equal levels of protein loading. Cells treated by HGPRT siRNAs were found to decrease HGPRT expression to an undetectable level in comparison to negative control siRNA or non-transfected samples (Figure 6). Additionally, no detectable difference was noticed between knockdowns of HGPRT using s6887 or s6888 siRNAs. Additional samples were transfected and then lysed at $24,48,72,96$, and 120 hours in order to determine the longevity of HGPRT knockdown. Substantial knockdown was found to occur at 24 hours and increased until 72 hours. From 72 hours to 120 , hours protein levels remained unchanged. The loading control of $\alpha$-tubulin did not exhibit substantial changes at any time point.

\section{Analyzing AS1411 Anti-Proliferative Activity in HGPRT Deficient Cells}

To determine if the anti-proliferative activity of AS1411 was dependent on HGPRT function, cells were transfected with HGPRT siRNAs, treated with drug, and then measured for levels of relative proliferation. A549 cells were transfected with HGPRT siRNA (s6887), a negative control siRNA, or left untransfected. After 48 hours, cells were treated with AS1411, CRO, or a water vehicle control for 72 hours before relative proliferation was measured. Results indicate that HGPRT knockdown caused no significant variance in AS1411 treatment when compared to controls (Figure 7). Furthermore, there was no significant variation between the vehicle control groups, implying that transfection was not inherently toxic to the cells. Additionally, treatment with $5 \mu \mathrm{M}$ AS1411 was found to significantly decrease proliferation $(p<0.01)$ when compared to $\mathrm{CRO}$ groups. 


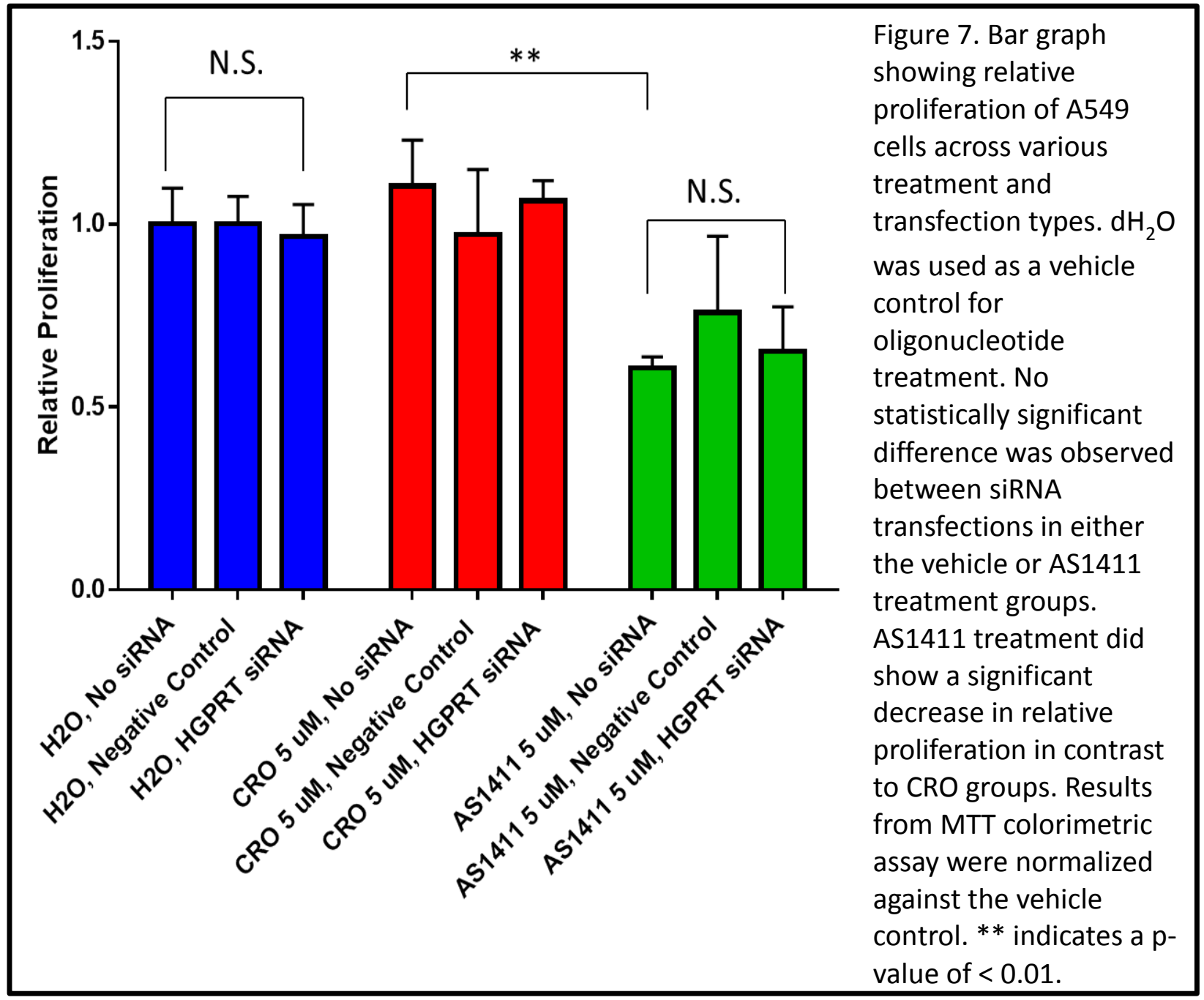

To further investigate if the anti-proliferative activity of AS1411 is dependent on HGPRT function, transfection parameters were altered to ensure HGPRT knockdown. In the first variation of the transfection protocol (Figure $8 \mathrm{~A}$ ), siRNA concentrations were increased from $20 \mathrm{nM}$ to $40 \mathrm{nM}$ in an attempt to create a more complete knockdown and determine if results were reproducible. Results from this trial demonstrate a high degree of similarity to previous findings. No significant variation was found in either the AS1411 or vehicle control, and AS1411 treated cells experienced a significant decrease in proliferation in comparison to CRO controls $(p<0.05)$. For the next experiment, the 
siRNA transfection time was reduced from 48 to 24 hours to ensure that the plate would be processed by MTT assay within 120 hours of transfection because previous findings did not study siRNA knockdown efficacy outside this range (Figure 6, B). Results (Figure 8, B) from this test had a high degree of similarity to the original findings (Figure

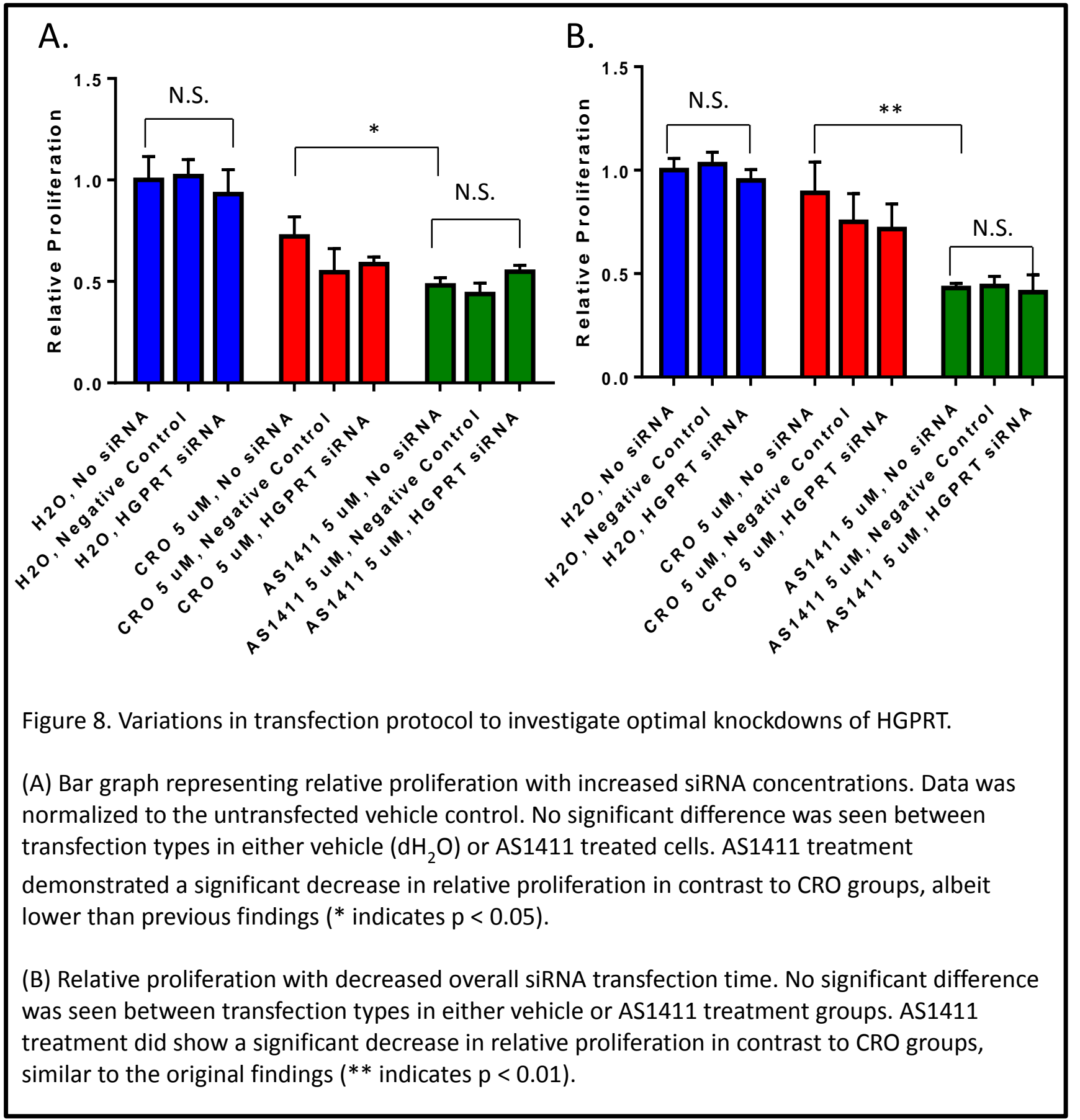

7). AS1411 and vehicle control treatment groups demonstrated no significant variation 
between transfection groups, and AS1411 was shown to have a significant decrease in proliferation when compared to the corresponding CRO treated cells $(p<0.05)$.

\section{Determining Optimal Conditions for GBPC Anti-Proliferative Effects in A549 and}

\section{MDA-MB-231 Cells}

A.

Effect of Guanosine on A549 Cell Proliferation

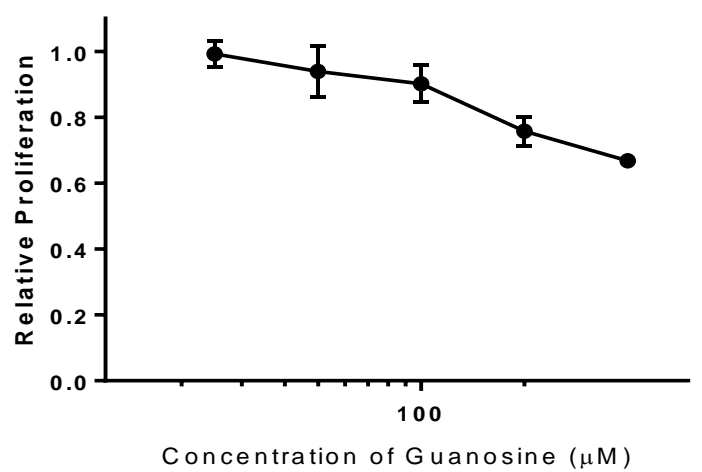

B.

Effect of Guanosine on M DA-MB-231 Cell Proliferation

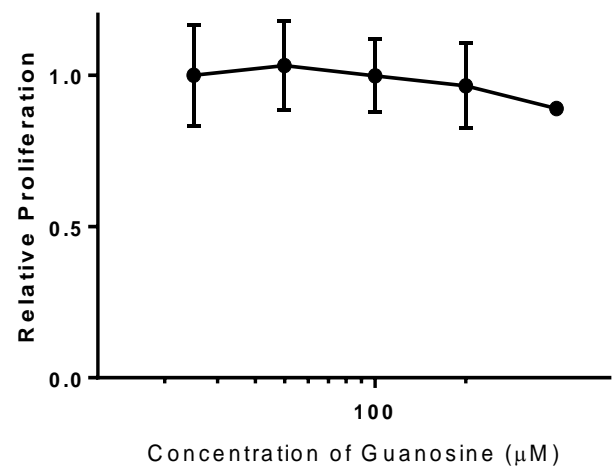

Figure 9. Comparing guanosine activity in A549 and MDA-MB231 cells.

(A) Graph showing relative proliferation of A549 cells treated with increasing concentrations of guanosine. A549 cells show a light response to guanosine treatment. Error bars represent one standard deviation from the mean.

(B) Graph showing relative proliferation of MDA-MB-231 cells treated with increasing concentrations of guanosine. MDA-MB-231 cells show a lessened response to guanosine treatment in comparison to A549 counterparts. Error bars represent one standard deviation from the mean.

As mentioned previously, guanine-based purine compounds (GBPCs), such as guanosine, have been shown to exhibit anti-proliferative effects in vitro [14].

Furthermore, their toxicity appears to be dependent on HGPRT function [15]. To determine if AS1411 activity is similar to that seen in cells treated with GBPCs, A549 and MDA-MB-231 cells were treated 25 to $400 \mu \mathrm{M}$ guanosine (Guo) ranging from 25 to $400 \mu \mathrm{M}$ for 72 hours before cell proliferation assays were performed. A549 cells 
demonstrated a dose-dependent response to Guo treatments (Figure 9, A); however, the anti-proliferative activity was not as substantial as seen with AS1411 at equivalent concentrations (Figure 4). A549 cells treated with $400 \mu \mathrm{M}$ Guo demonstrated a relative proliferation of $60 \%$ compared to the control, whereas MDA-MB-231 cells at an identical concentration only demonstrated a decrease to $80 \%$ when compared to the control (Figure 9).

\section{GBPC Anti-Proliferative Activity in HGPRT Deficient A549 and MDA-MB-231 Cells}

After determining the responsiveness of A549 and MDA-MB-231 cell lines to guanosine, further experiments sought to determine if siRNA knockdown of HGPRT would decrease the anti-proliferative activity caused by guanosine. Previous literature has demonstrated that the loss of HGPRT resulted in the loss of anti-proliferative activity upon exposure to guanosine [15]. If siRNA knockdown of HGPRT decreases the activity of Guo, then it would suggest that the transfection can effectively reduce HGPRT activity. However, if transfection is unable to significantly affect anti-proliferative activity in Guo treatment, then it would suggest that the knockdown is not thorough enough to fully negate HGPRT enzymatic activity. A549 and MDA-MB-231 cells were transfected with siRNAs targeting HGPRT, a negative control siRNA, or left untransfected. After 48 hours, cells were treated with increasing concentrations of Guo, AS1411, or vehicle for 72 hours before relative proliferation was measured. Results in A549 cells indicate that cells treated with a vehicle control and transfected with HGPRT 
siRNA displayed a decrease in proliferation of 35\% in comparison to those transfected with a negative control siRNA or left untransfected (Figure 10). A dose-dependent response to Guo treatment was seen across the A549 cells, with $400 \mu \mathrm{M}$ treatments reducing relative proliferation by $40-50 \%$ depending on transfection type. AS1411 treated groups, however, still exhibited greater drops in proliferation of $60-65 \%$. No significant difference was seen between transfection types in cells treated with $50 \mu \mathrm{M}$,

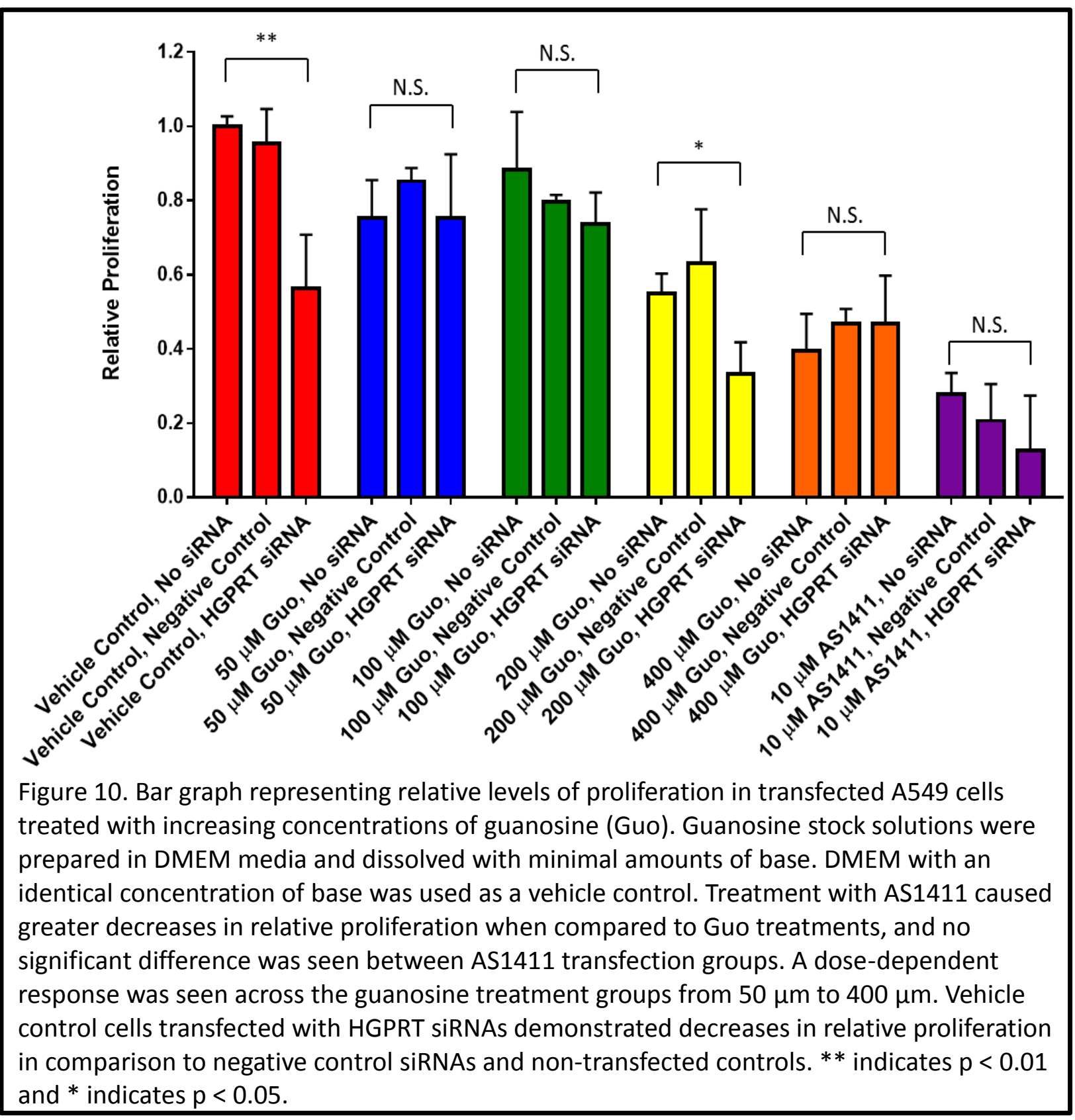


$100 \mu \mathrm{M}$, or $400 \mu \mathrm{M}$ Guo. Although there was a statistically significant difference seen in $200 \mu \mathrm{M}$ Guo groups $(p=0.048)$, replication would be needed to confirm the results.

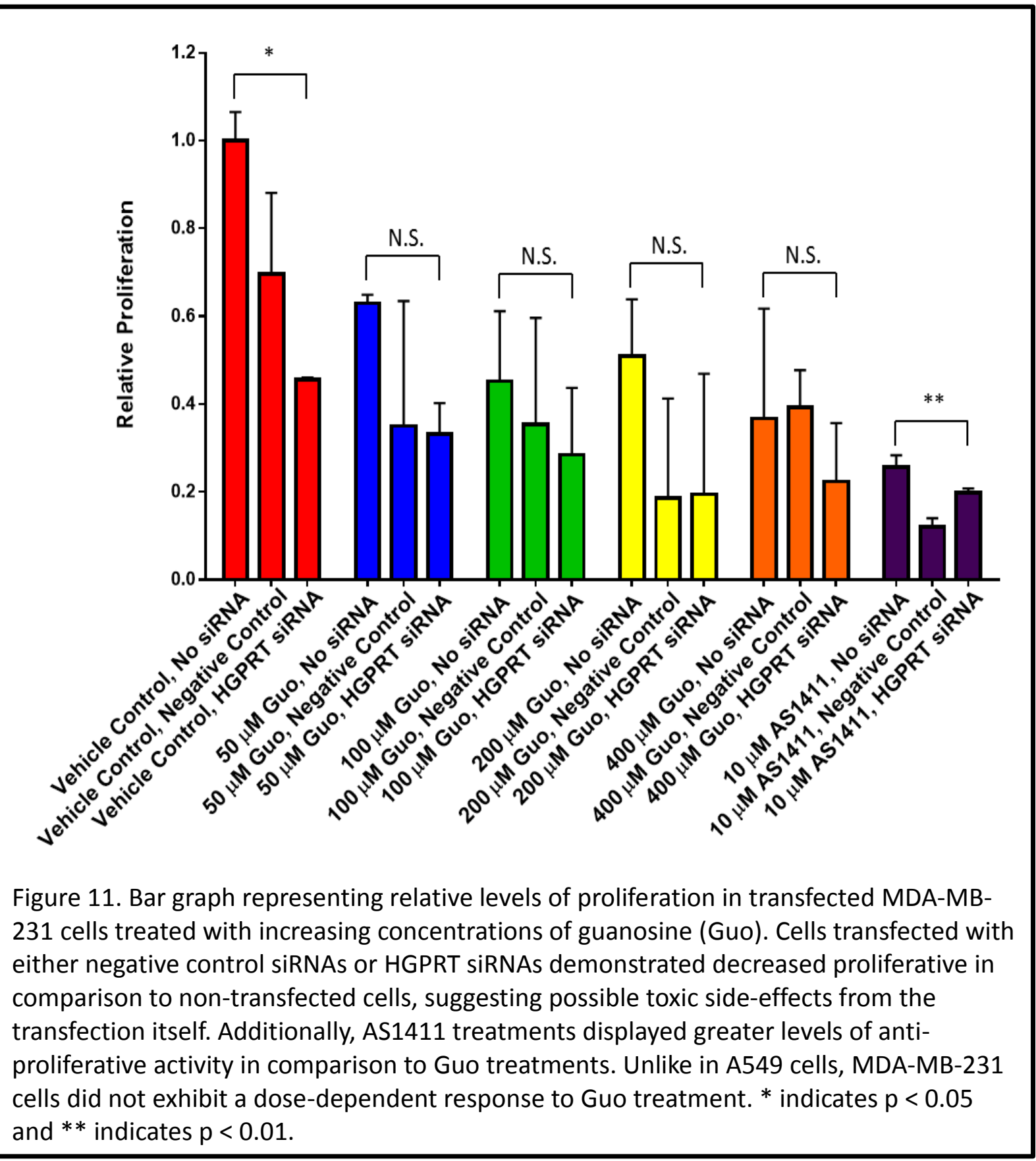

Results in MDA-MB-231cells did not indicate a strong response to Guo treatment; however, cells transfected with either HGPRT siRNAs or negative control 
siRNAs demonstrated similar levels of relative proliferation (Figure 11). This suggests possible toxic side-effects from the lipofectamine. Non-transfected cells treated with Guo consistently demonstrated a decrease in relative proliferation of $\sim 40 \%$. These outcomes are consistent with the findings of the dose response curve in which A549 cells demonstrated a greater sensitivity to Guo treatment than MDA-MB-231 cells (Figure 9). Again, cells transfected with HGPRT siRNAs and treated with the vehicle demonstrated significant decreases in proliferation when compared to non-transfected cells, suggesting a toxic side effect with the loss of HGPRT function.

\section{Evaluating GBPC and AS1411 Anti-Proliferative Activity in 6-Thioguanine Resistant Cells}

A549 cells were incubated with concentrations of 6-thioguanine (TG) in order to select for HGPRT mutant cells. Inside the cell, TG acts as an analog to guanine and can be recycled by HGPRT and then incorporated into the DNA [20]. This incorporation typically results in strand breakage and eventual cellular death; however, the loss of HGPRT activity can remove the toxic effects of TG [21]. Based on a previous protocol [22], HGPRT mutants were selected by increasing treatments of 6-thioguanine and then treated with increasing concentrations of Guo and AS1411. A549 cells incubated in TG and wildtype cells were plated for 24 hours, treated with a vehicle control, Guo, or AS1411 for 72 hours, and then relative proliferation was measured. Although no significant difference in proliferation was seen in the vehicle controls, TG treated cells did show increased proliferation across three of the four Guo treatments $(50 \mu \mathrm{M}, 100$ $\mu \mathrm{M}$, and $200 \mu \mathrm{M})$. Additionally, TG treated cells demonstrated greater levels of 
proliferation when treated with AS1411 in comparison to wildtype A549 cells (Figure 12).

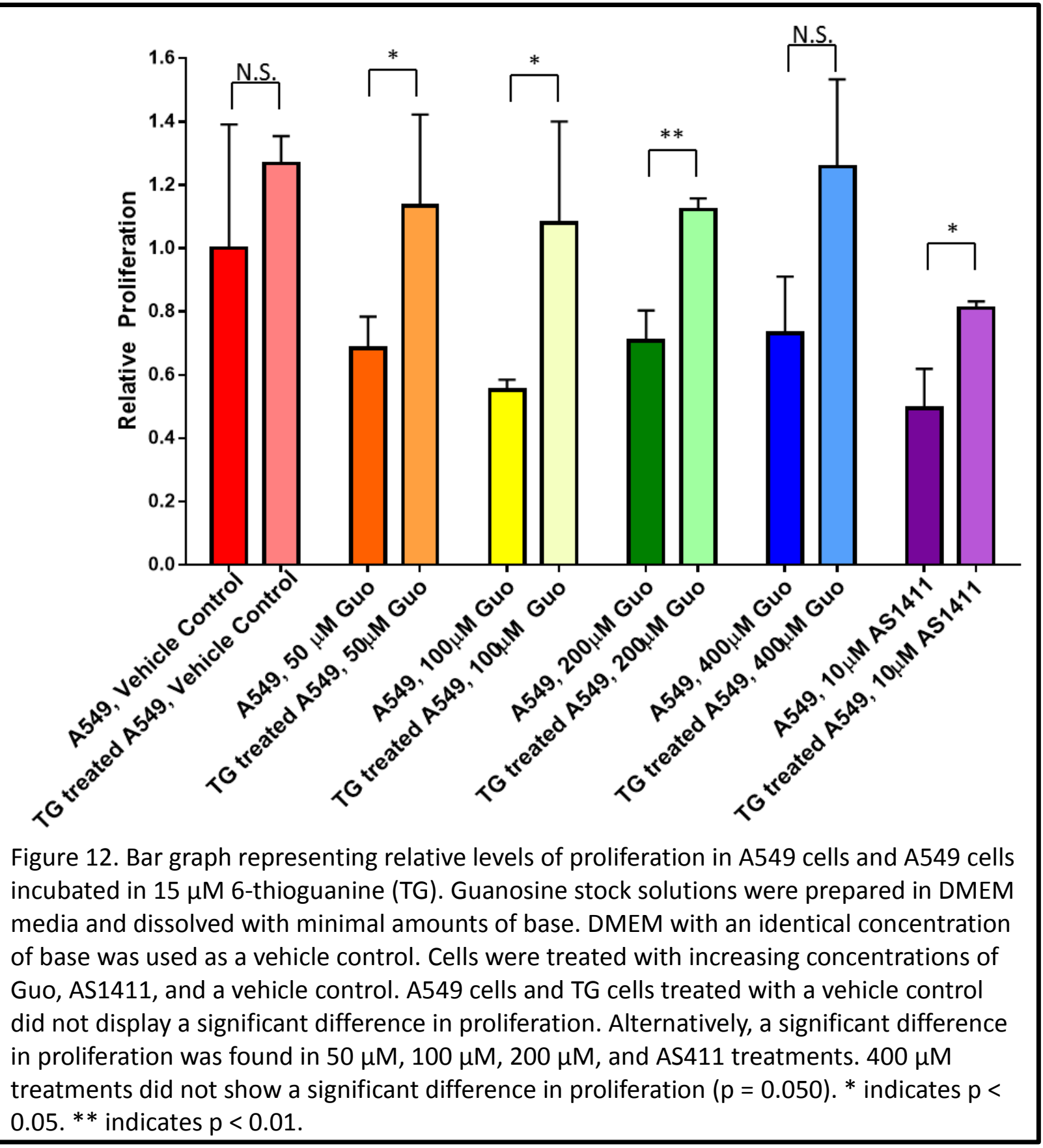




\section{Discussion}

Initial experiments were conducted to establish a baseline concentration for AS1411 anti-proliferative activity in A549 cells and to ensure that HGPRT expression could be knocked down by transfection with siRNAs. Investigations into the efficacy of AS1411 treatments in A549 cells demonstrated a Gl 50 for AS1411 of $1.5 \mu \mathrm{M}$ (Figure 4). These findings are congruent with previous findings that cited the Gl 50 range for AS1411 in A549 cells being approximately $2 \mu \mathrm{M}$ [23]. Immunoblots of transfected cells were found to decrease expression of HGPRT when treated with either HGPRT siRNA (s6887 \& s6888). This knockdown effect was seen to begin at 24 hours and last through 120 hours. No difference in protein knockdown was found between the HGPRT siRNAs s6887 \& s6888, so only s6887 was used for transfections. Non-transfected cells were used as a baseline for HGPRT expression, and cells transfected with a negative control siRNA were used to gauge whether or not transfection with lipofectamine altered protein expression. Cells transfected with negative control siRNAs and non-transfected cells were found to have similar levels of protein expression implying that transfection with lipofectamine did not alter HGPRT expression. Expression of $\alpha$-tubulin was measured to ensure that equal amounts of protein were loaded during the immunoblot process. The loading controls did not exhibit any irregularities in expression that would discredit previous findings.

After the Gl 50 of AS1411 in A549 cells and reliable HGPRT knockdown were established, A549 cells were transfected and then treated with AS1411 to test if HGPRT activity was necessary for AS1411 anti-proliferative effects. CRO (cytosine rich oligonucleotide) treatments in equal concentrations to AS1411 counterparts were used 
to determine if oligonucleotide treatment decreased relative proliferation as seen in previous studies with AS1411 [24]. Additionally, water was used as a vehicle control. In line with previous findings, AS1411 displayed strong anti-proliferative effects compared to vehicle and $\mathrm{CRO}$ control treatments. Transfected vehicle control treatments did not exhibit decreased proliferation, suggesting that transfection was not toxic to cells. Additionally, CRO treatments did not differ significantly to vehicle control treatments implying that non-specific oligonucleotide treatment was not toxic to cells. Levels of proliferation did not vary between cells treated with AS1411 and transfected with HGPRT siRNAs, negative control siRNAs, or left untransfected.

These results could imply multiple outcomes. Since the downregulation of HGPRT seemingly did not alter AS1411 anti-proliferative activity, it is possible that the enzyme is not a necessary element for AS1411 activity, possibly because degradation is not an essential step in the drug pathway. Alternatively, transfections with HGPRT siRNAs may have knocked down most, but not all of the HGPRT in a cell. In this scenario, it is possible that a small amount of residual HGPRT was able to preserve the activity of AS1411.

Additional tests were performed in order to explore the latter possibility. A549 cells were transfected and treated with doubled concentrations of siRNA for comparison with the previous findings to determine if initial concentrations had produced an incomplete knockdown. The findings were congruent with previous results. Cells treated with AS1411 and water did not show any significant change in relative proliferation across transfection type, and AS1411-treated cells demonstrated lower relative proliferation when compared to either water or CRO groups (Figure $8 \mathrm{~A}$ ). These results 
suggest that initial concentrations of siRNA were adequate to knockdown further HGPRT mRNA translation. Additionally, tests were performed in which transfection time was reduced from 48 to 24 hours to ensure that proliferation was being measured inside the previously established 120 hour window of HGPRT knockdown. Again, results were consistent with the original findings. AS1411 treated cells demonstrated decreased proliferation when compared to CRO or water treated cells. Transfections did not significantly alter levels of relative proliferation in either the $\mathrm{CRO}$ or water groups. These results indicate that data from initial time points were accurately reflected cellular proliferation from treatment and knockdown.

Although consistent, this transfection data does not fully answer our previous question regarding siRNA knockdown of HGPRT. Was there no effect with AS1411 treatment because the compound works by a non-HGPRT dependent mechanism, or because a residual population of HGPRT exists to preserve anti-proliferative activity? To answer this, we decided to test our siRNA HGPRT knockdown against GBPC antiproliferative activity. As mentioned previously, GBPCs have antagonistic effects on cellular growth, and these effects are known to be dependent on HGPRT activity [14 \& 15]. If $A S 1411$ and GBPC activity works by an analogous mechanism due to similar chemical composition and exhibited anti-proliferative effects in cancer cell lines, this would mean that HGPRT siRNA transfections would affect the anti-proliferative effects of both compounds in a similar manner. If HGPRT knockdowns are unable to cause a change in GBPC activity, it would imply that siRNAs were unable to effectively reduce HGPRT activity and imply that residual pools of HGPRT are preserving AS1411 and GBPC function. Conversely, if the GBPCs do demonstrate decreases in activity in cells 
with decreased levels of HGPRT, it would imply that HGPRT siRNAs are able to efficiently knockdown HGPRT activity and that AS1411 works by a non-HGPRT mechanism.

To test this hypothesis, the anti-proliferative effects of GBPCs in our cell lines needed to be established. A549 and MDA-MB-231 cells were treated with concentrations of Guo ranging from $25-400 \mu \mathrm{M}$. These concentrations were chosen in hopes of developing a dose-dependent response curve and to mimic concentrations of AS1411 used previously. AS1411 contains approximately 20 guanine bases, meaning that a $10 \mu \mathrm{M}$ treatment of $\mathrm{AS} 1411$ would be equivalent to a $200 \mu \mathrm{M}$ treatment of Guo. A549 and MDA-MB-231 cells both exhibited a response to high concentrations of Guo, but results were not as drastic as that seen in AS1411 treatments. Additionally, the cells demonstrated differing levels of response to the treatment, A549 cells treated with 400 $\mu \mathrm{M}$ of Guo decreased in proliferation by approximately $40 \%$ when compared to the vehicle, whereas the decrease in MDA-MB-231 cells was closer to $20 \%$ (Figure 9). Treatments of $200 \mu \mathrm{M}$ Guo did not decrease proliferation in either cell line to that which was seen in $10 \mu \mathrm{M}$ treatments of AS1411 (Figure 5).

With the reactivity of each cell line to GBPCs established, the activity of the Guo was then tested after transfection with HGPRT siRNAs with the same time points and protocols as previous AS1411 studies. Treatments of Guo in non-transfected cells followed previous patterns. A549 cells demonstrated a dose dependent response to Guo treatment, but MDA-MB-231 cells did not exhibit a strong dose dependent response. Furthermore, treatment with AS1411 caused greater decreases in 
proliferation than Guo, which is consistent with previous dose-response curves in AS1411 (Figure 5) and Guo (Figure 9). Unfortunately, results in MDA-MB-231 cells demonstrated decreases in proliferation in both negative control siRNA and HGPRT siRNA transfections suggesting a toxic side-effect of lipofectamine in MDA-MB-231 cells at that concentration. Of the eight Guo treatment groups between the two cells types, seven did not show significant differences between non-transfected, negative control siRNA transfections, and HGPRT siRNA transfections. One group displayed a statistically significant difference in proliferation among the transfection types, A549, $200 \mu \mathrm{M}$ Guo. In the majority of groups, HGPRT knockdown was unable to significantly alter Guo anti-proliferative activity. Therefore, these experiments have not determined whether or not the activity of AS1411 is the same as GBPCs.

Expression knockdown via transfected siRNAs operates by engineering siRNAs with complimentary sequences to the mRNA of interest and delivering them into the cell via cationic liposomes [25]. Once inside the cell, the siRNAs integrate into the multiprotein RNAi induced silencing complex (RISC), which can target and degrade the desired mRNAs [26]. Although this process will knockdown the expression of a particular protein by a considerable amount, it will not target the preexisting proteins within the cell. For proteins with short half-lives, this is not problematic for the experimental procedure; however, some proteins can have half-lives of over 100 hours [27]. Additionally, the proteins of cells in stressed environments where cellular division has been slowed or stopped can actually increase their half-lives [28]. HGPRT has a half-life of over 48 hours [29], which may be extended during periods of stress caused by treatments of AS1411 or Guo. Previous results demonstrate an incomplete ability to 
prevent HGPRT activity, which may be due in part to residual HGPRT inside the cell that is not silenced by siRNA transfection.

In order to test the activity of these compounds in a HGPRT deficient environment, A549 cells were incubated in TG in order to select for HGPRT mutant cells. As mentioned previously, TG is toxic to cells with functioning HGPRT. HGPRT can transfer a phosphoribosyl group onto TG, allowing it to be incorporated into the DNA and cause strand breakages [20 \& 21]. The employed protocol was based on previous work [22] that demonstrates how cells can be selected for HGPRT mutations for mutagenesis studies. After establishing A549 cell lines that could consistently grow in $15 \mu \mathrm{M} \mathrm{TG}$, their proliferation in GBPCs and AS1411 were measured against wild type A549 cells. A statistically significant increase in proliferation was seen across multiple Guo treatments as well as in AS1411 treatments for cells incubated in 6-thioguanine over wild type counterparts. Although the mutant cells were not examined by a genomic assay, their ability to grow and proliferate while being incubated in TG suggests that the cells did not have a functioning copy of HGPRT. Coupled with the attenuated responses of HGPRT mutants to GBPC/AS1411 treatment, this suggests that HGPRT activity is vital for the activity of GBPCs and AS1411. Furthermore, the anti-proliferative activity of both AS1411 and GBPCs may likely operate by a similar, HGPRT dependent mechanism. While the results are promising, these experiments have a number of limitations. A lack of genomic testing of the TG treated cells to confirm mutations in the HPRT1 gene means that their TG resistance may not have come from a non HGPRTrelated mechanism. Although the vehicle control treatments in TG treated cells and wildtype cells did not demonstrate significant difference in proliferation, this result could 
have been skewed by the large error bar seen in the vehicle treated wildtype cells. Additionally, the experiment lacked a CRO control to ensure that differences in proliferation can be attributed to AS1411 and Guo anti-proliferative effects and not simply to physiological differences in the two cell lines due to TG related stress. Replication of the experiment with these additional controls would be necessary to confirm previous results.

\section{Future Directions}

Although these results suggest a similar mechanism for AS1411 and GBPCs, more work is necessary to confirm this hypothesis. Replication of these experiments in additional cell lines that are more responsive to Guo treatment would help to strengthen this results. Additionally, genomic testing of TG-incubated A549 cells would be crucial to confirm mutations in the HPRT1 gene compromised the function of HGPRT. Moreover, a CRISPR/Cas9 system could be used to create an HGPRT deficient cell line analogous to HGPRT mutants to further confirm that previous results were due to the loss of HGPRT function. 


\section{References}

1. American Cancer Society. Cancer Facts \& Figures 2017.

2. "Chemotherapy Side Effects." American Cancer Society. N.p., n.d. Web. 08 Mar. 2017.

3. "What about radiation side effects?" American Cancer Society. N.p., n.d. Web. 08 Mar. 2017.

4. Mandal, MD Dr Ananya. "What is an Oligonucleotide?" News-Medical.net. N.p., 10 Sept. 2014. Web. 08 Mar. 2017.

5. Bates, Paula J., Damian A. Laber, Donald M. Miller, Shelia D. Thomas, and John O. Trent. "Discovery and Development of the G-rich Oligonucleotide AS1411 as a Novel Treatment for Cancer." Experimental and Molecular Pathology 86.3 (2009): 151-64.

6. M.M. Dailey, M.C. Miller, P.J. Bates, A.N. Lane, J.O. Trent Resolution and characterization of the structural polymorphism of a single quadruplex-forming sequence Nucleic Acids Res., 38 (2010), pp. 4877-4888

7. Ma, Dik-Lung, Chung-Hang Leung, Daniel Shiu-Hin Chan, Hai-Jing Zhong, Hong-Zhang He, Ka-Ho Leung, and Victor Pui-Yan Ma. Structure-Based Approaches Targeting Oncogene Promoter G-Quadruplexes. N.p.: INTECH Open Access Publisher, 2013. Print.

8. Bates, Paula J., Elsa M. Reyes-Reyes, Mohammad T. Malik, Emily M. Murphy, Martin G. O'toole, and John O. Trent. "G-quadruplex oligonucleotide AS1411 as a cancer-targeting agent: Uses and mechanisms." Biochimica et Biophysica Acta (BBA) - General Subjects (2016): n. pag. Web.

9. Rosenberg, Jonathan E., Richard M. Bambury, Eliezer M. Van Allen, Harry A. Drabkin, Primo N. Lara, Andrea L. Harzstark, Nikhil Wagle, Robert A. Figlin, Gregory W. Smith, Levi A. Garraway, Toni Choueiri, Fredrik Erlandsson, and Damian A. Laber. "A Phase II Trial of AS1411 (a Novel Nucleolin-targeted DNA Aptamer) in Metastatic Renal Cell Carcinoma." Invest New Drugs Investigational New Drugs 32.1 (2013): 178-87. Web.

10. Reyes-Reyes, E. Merit, Francesca R. Šalipur, Mitra Shams, Matthew K. Forsthoefel, and Paula J. Bates. "Mechanistic studies of anticancer aptamer AS1411 reveal a novel role for nucleolin in regulating Rac1 activation." Molecular Oncology 9.7 (2015): 1392-405. Web.

11. Trabbic, Christopher J., Heather M. Dietsch, Evan M. Alexander, Peter I. Nagy, Michael W. Robinson, Jean H. Overmeyer, William A. Maltese, and Paul W. Erhardt. "Differential Induction of Cytoplasmic Vacuolization and Methuosis by Novel 2-Indolyl-Substituted Pyridinylpropenones." ACS Medicinal Chemistry Letters 5.1 (2014): 73-77. Web. 
12. Maltese, William A., and Jean H. Overmeyer. "Methuosis." The American Journal of Pathology 184.6 (2014): 1630-642. Web.

13. Yechezkel, Sidi, Jerry Hudson, and Beverly Mitchell. "Effects of Guanine Ribonucleotide Accumulation on the Metabolism and Cell Cycle of Human Lymphoid Cells." Cancer Research 45.10 (1985): n. pag. Web. 23 Mar. 2017.

14.Zhang, Nan, Tao Bing, Xiangjun Liu, Cui Qi, Luyao Shen, Linlin Wang, and Dihua Shangguan. "Cytotoxicity of Guanine-based Degradation Products Contributes to the Anti-proliferative Activity of Guanine-rich Oligonucleotides." Chem. Sci. 6.7 (2015): 3831-838. Web.

15. Garozzo, Roberta, Maria Angela Sortino, Carlo Vancheri, and Daniele Filippo Condorelli. "Anti-proliferative Effects Induced by Guanine-based Purines Require Hypoxanthine-guanine Phosphoribosyltransferase Activity." Biological Chemistry 391.9 (2010): n. pag. Web.

16. Ansari, Md Yousuf, Asif Equbal, Manas Ranjan Dikhit, Rani Mansuri, Sindhuprava Rana, Vahab Ali, Ganesh Chandra Sahoo, and Pradeep Das. "Establishment of correlation between in-silico and in-vitro test analysis against Leishmania HGPRT to inhibitors." International Journal of Biological Macromolecules 83 (2016): 78-96. Web.

17. National Institutes of Health. U.S. Department of Health and Human Services, n.d. Web. 08 Mar. 2017.

18. Morgan, David M. L. "Tetrazolium (MTT) Assay for Cellular Viability and Activity." Polyamine Protocols (n.d.): 179-84. Web.

19. Bates, P. J., J. B. Kahlon, S. D. Thomas, J. O. Trent, and D. M. Miller. "Antiproliferative Activity of G-rich Oligonucleotides Correlates with Protein Binding." Journal of Biological Chemistry 274.37 (1999): 26369-6377. Web.

20. Albertini, Richard J., J. Patrick O'neill, Janice A. Nicklas, Nicholas H. Heintz, and Philip C. Kelleher. "Alterations of the hprt gene in human in vivo-derived 6thioguanine-resistant T lymphocytes." Nature 316.6026 (1985): 369-71. Web.

21. Choudhary, Rashmi, Dmitry Baturin, Susan Fosmire, Brian Freed, and Christopher C. Porter. "Knockdown of HPRT for Selection of Genetically Modified Human Hematopoietic Progenitor Cells." PLoS ONE 8.3 (2013): n. pag. Web.

22. Monnat, Ray. "Protocol for HPRT mutagenesis analyses

." Http://depts.washington.edu. University of Washington, Apr. 2000. Web. $<$ http://depts.washington.edu/monnatws/pdf/HPRT_protocols_042009.pdf>.

23. Ireson, C. R., and L. R. Kelland. "Discovery and development of anticancer aptamers." Molecular Cancer Therapeutics 5.12 (2006): 2957-962. Web.

24. Reyes-Reyes, E. M., Y. Teng, and P. J. Bates. "A New Paradigm for Aptamer Therapeutic AS1411 Action: Uptake by Macropinocytosis and Its Stimulation by a Nucleolin-Dependent Mechanism." Cancer Research 70.21 (2010): 8617-629. Web. 
25. "SiRNA - Applications." Applications - siRNA / GE Dharmacon. N.p., n.d. Web. 08 Mar. 2017.

26. "RNAi Explorer." Gene Link : What is RNAi and Introductions. N.p., n.d. Web. 08 Mar. 2017.

27. Schwanhäusser, Björn, Dorothea Busse, Na Li, Gunnar Dittmar, Johannes Schuchhardt, Jana Wolf, Wei Chen, and Matthias Selbach. "Global quantification of mammalian gene expression control." Nature 473.7347 (2011): 337-42. Web.

28. Eden, E., N. Geva-Zatorsky, I. Issaeva, A. Cohen, E. Dekel, T. Danon, L. Cohen, A. Mayo, and U. Alon. "Proteome Half-Life Dynamics in Living Human Cells." Science 331.6018 (2011): 764-68. Web.

29. Friedmann, Theodore. Molecular genetic medicine. Vol. 2. San Diego: Academic Press, 1992. Print. 